\title{
Existence and multiplicity of solutions for a fourth-order elliptic equation
}

Fanglei Wang ${ }^{1 *}$ and Yukun $\mathrm{An}^{2}$

\author{
* Correspondence: wang- \\ fanglei@hotmail.com \\ ${ }^{1}$ College of Science, Hohai \\ University, Nanjing, 210098, P. R. \\ China \\ Full list of author information is \\ available at the end of the article
}

\section{Abstract}

This article is concerned with the existence and multiplicity of nontrival solutions for a fourth-order elliptic equation

$$
\begin{cases}\Delta^{2} u-M\left(\int_{\Omega}|\nabla u|^{2} d x\right) \Delta u=f(x, u), & \text { in } \Omega, \\ u=\Delta u=0, & \text { on } \partial \Omega\end{cases}
$$

by using the mountain pass theorem.

Keywords: fourth-order elliptic equation, nontrivial solutions, mountain pass theorem

\section{Introduction}

In this article we study the existence of nontrivial solutions for the fourth-order boundary value problem

$$
\begin{cases}\Delta^{2} u-M\left(\int_{\Omega}|\nabla u|^{2} d x\right) \Delta u=f(x, u), & \text { in } \Omega, \\ u=\Delta u=0, & \text { on } \partial \Omega\end{cases}
$$

where $\Omega \subset R^{N}$ is a bounded smooth domain, $f: \Omega \times R \rightarrow R$ and $M: R \rightarrow R$ are continuous functions. The existence and multiplicity results for Equation (1) are considered in [1-3] by using variational methods and fixed point theorems in cones of ordered Banach space with space dimension is one.

On the other hand, The four-order semilinear elliptic problem

$$
\begin{cases}\Delta^{2} u+c \Delta u=f(x, u), & \text { in } \Omega, \\ u=\Delta u=0, & \text { on } \partial \Omega\end{cases}
$$

arises in the study of traveling waves in a suspension bridge, or the study of the static deflection of an elastic plate in a fluid, and has been studied by many authors, see [4-10] and the references therein.

Inspired by the above references, the object of this article is to study existence and multiplicity of nontrivial solution of a fourth-order elliptic equation under some conditions on the function $M(t)$ and the nonlinearity. The proof is based on the mountain pass theorem, namely,

Lemma 1.1. Let $E$ be a real Banach space, and $I \in C^{1}(E, R)$ satisfy $(P S)$-condition. Suppose

\section{Springer}

(C) 2012 Wang and An; licensee Springer. This is an Open Access article distributed under the terms of the Creative Commons Attribution License (http://creativecommons.org/licenses/by/2.0), which permits unrestricted use, distribution, and reproduction in any medium, provided the original work is properly cited. 
(1) There exist $\rho>0, \alpha>0$ such that

$$
\left.I\right|_{\partial B_{\rho}} \geq I(0)+\alpha
$$

where $B_{p}=\{u \in E \mid\|u\| \leq \rho\}$.

(2) There is an $e \in E$ and $\|e\|>\rho$ such that

$$
I(e) \leq I(0)
$$

Then $I(u)$ has a critical value $c$ which can be characterized as

$$
C=\inf _{\gamma \in \Gamma} \max _{u \in \gamma([0,1])} I(u)
$$

where $\Gamma=\{\gamma \in C([0,1], E) \mid \gamma(0)=0, \gamma(1)=e\}$.

The article is organized as follows: Section 2 is devoted to giving the main result and proving the existence of nontrivial solution of Equation (1). In Section 3, we deal with the multiplicity results of Equation (1) whose nonlinear term is asymptotically linear at both zero and infinity

\section{Main result I}

Theorem 2.1. Assume the function $M(t)$ and the nonlinearity $f(x, t)$ satisfying the following conditions:

(H1) $M(t)$ is continuous and satisfies

$$
M(t)>m_{0}, \quad \forall t>0,
$$

for some $m_{0}>0$. In addition, that there exist $m^{\prime}>m_{0}$ and $t_{0}>0$, such that

$$
M(t)=m^{\prime}, \quad \forall t>t_{0}
$$

(H2) $f(x, t) \in C(\Omega \times R) ; f(x, t) \equiv 0, \forall x \in \Omega, t \leq 0, f(x, t) \geq 0, \forall x \in \Omega, t>0$;

(H3) $|f(x, t)| \leq a(x)+b|t|^{p}, \forall t \in R$ and a.e. $x$ in $\Omega$, where $a(x) \in L^{q}(\Omega), b \in R$

and $1<p<\frac{N+4}{N-4}$ if $N>4$ and $1<p<\infty$ if $N \leq 4$ and $\frac{1}{q}+\frac{1}{p}=1$;

(H4) $f(x, t)=o(|t|)$ as $t \rightarrow 0$ uniformly for $x \in \Omega$;

(H5) There exists a constant $\Theta>2$ and $\mathrm{R}>0$, such that

$$
\Theta F(x, s) \leq s f(x, s), \quad \forall|s| \geq R
$$

Then Equation (1) has at least one nonnegative solution.

Let $\Omega \subset R^{N}$ be a bounded smooth open domain, $\mathbf{H}=\mathbf{H}^{2}(\Omega) \bigcap \mathbf{H}_{0}^{1}(\Omega)$ be the Hilbert space equipped with the inner product

$$
(u, v)-\int_{\Omega}(\Delta u \Delta v+\nabla u \nabla v) d x
$$


and the deduced norm

$$
\|u\|^{2}=\int_{\Omega}|\Delta u|^{2} d x+\int_{\Omega}|\nabla u|^{2} d x .
$$

Let $\lambda_{1}$ be the positive first eigenvalue of the following second eigenvalue problem

$$
\begin{cases}-\Delta v=\lambda v, & \text { in } \Omega \\ v=0, & \text { on } \partial \Omega\end{cases}
$$

Then from [4], it is clear to see that $\Lambda_{1}=\lambda_{1}\left(\lambda_{1}-c\right)$ is the positive first eigenvalue of the following fourth-order eigenvalue problem

$$
\begin{cases}\Delta^{2} u+c \Delta u=\lambda u, & \text { in } \Omega, \\ \Delta u=u=0, & \text { on } \partial \Omega\end{cases}
$$

where $c<\lambda_{1}$. By Poincare inequality, for all $u \in \mathbf{H}$, we have

$$
\|u\|^{2} \geq \Lambda_{1}\|u\|_{L^{2}}^{2} .
$$

A function $u \in \mathbf{H}$ is called a weak solution of Equation (1) if

$$
\int_{\Omega} \Delta u \Delta v d x+M\left(\int_{\Omega}|\nabla u|^{2} d x\right) \int_{\Omega} \nabla u \nabla v d x=\int_{\Omega} f(x, u) v d x
$$

holds for any $v \in \mathbf{H}$. In addition, we see that weak solutions of Equation (1) are critical points of the functional $I: \mathbf{H} \rightarrow R$ defined by

$$
I(u)=\frac{1}{2} \int_{\Omega}|\Delta u|^{2} d x+\frac{1}{2} \widehat{M}\left(\int_{\Omega}|\nabla u|^{2} d x\right)-\int_{\Omega} F(x, u) d x,
$$

where $\widehat{M}(t)=\int_{0}^{t} M(s) d s$ and $F(x, t)=\int f(x, t) d t$. Since $M$ is continuous and $f$ has subcritical growth, the above functional is of class $C^{1}$ in $\mathbf{H}$. We shall apply the famous mountain pass theorem to show the existence of a nontrivial critical point of functional $I(u)$.

Lemma 2.2. Assume that (H1)-(H5) hold, then $I(u)$ satisfies the (PS)-condition.

Proof. Let $\left\{u_{n}\right\} \subset \mathbf{H}$ be a (PS)-sequence. In particular, $\left\{u_{n}\right\}$ satisfies

$$
I\left(u_{n}\right) \rightarrow C, \quad \text { and } \quad\left\langle I^{\prime}\left(u_{n}\right), u_{n}\right\rangle \rightarrow 0 \quad \text { as } n \rightarrow \infty .
$$

Since $f(x, t)$ is sub-critical by (H3), from the compactness of Sobolev embedding and, following the standard processes we know that to show that $I$ verifies $(P S)$-condition it is enough to prove that $\left\{u_{n}\right\}$ is bounded in $\mathbf{H}$. By contradiction, assume that $\left\|u_{n}\right\| \rightarrow$ $+\infty$.

Case I. If $\int_{\Omega}\left|\nabla u_{n}\right|^{2} d x$ is bounded, $\int_{\Omega}\left|\Delta u_{n}\right|^{2} d x \rightarrow+\infty$. We assume that there exist a constant $K>0$ such that $\int_{\Omega}\left|\nabla u_{n}\right|^{2} d x \leq K$. By (H1), it is easy to obtain that $\tilde{m}=\max _{t \in[0, K]} M(t)>m_{0}$. Set $l_{1}=\min \left\{1, m_{0}\right\}, l_{2}=\max \{1, \tilde{m}\}$. Then, from 
(H1), (H3), and (H5), we have

$$
\begin{aligned}
I\left(u_{n}\right)-\frac{l_{1}}{2 l_{2}} I^{\prime}\left(u_{n}\right) u_{n}= & \frac{1}{2} \int_{\Omega}\left|\Delta u_{n}\right|^{2} d x+\frac{1}{2} \widehat{M}\left(\int_{\Omega}\left|\nabla u_{n}\right|^{2} d x\right)-\int_{\Omega} F\left(x, u_{n}\right) d x \\
& -\frac{l_{1}}{2 l_{2}}\left(\int_{\Omega}\left|\Delta u_{n}\right|^{2} d x+M\left(\int_{\Omega}\left|\nabla u_{n}\right|^{2} d x\right) \int_{\Omega}\left|\nabla u_{n}\right|^{2} d x\right) \\
& +\frac{l_{1}}{2 l_{2}} \int_{\Omega} f\left(x, u_{n}\right) u_{n} d x \\
\geq & \frac{1}{2} l_{1}\left\|u_{n}\right\|^{2}+\int_{\Omega}\left[\frac{l_{1}}{2 l_{2}} f\left(x, u_{n}^{+}\right) u_{n}-F\left(x, u_{n}^{+}\right)\right] d x \\
\geq & \frac{1}{2} l_{1}\left\|u_{n}\right\|^{2}+\int_{\left\|u_{n}\right\| \geq R}\left[\frac{l_{1}}{2 l_{2}} f\left(x, u_{n}^{+}\right) u_{n}^{+}-F\left(x, u_{n}^{+}\right)\right] d x-C_{1} \\
\geq & \frac{1}{2} l_{1}\left\|u_{n}\right\|^{2}+\frac{l_{1}}{2 l_{2}} \int_{\left\|u_{n}\right\| \geq R}\left[f\left(x, u_{n}^{+}\right) u_{n}^{+}-\frac{2 l_{2}}{l_{2}} F\left(x, u_{n}^{+}\right)\right] d x-C_{1} \\
\geq & \frac{1}{2} l_{1}\left\|u_{n}\right\|^{2}+\frac{l_{1}}{2 l_{2}} \int_{\left\|u_{n}\right\| \geq R}\left[f\left(x, u_{n}^{+}\right) u_{n}^{+}-\Theta F\left(x, u_{n}^{+}\right)\right] d x-C_{1} .
\end{aligned}
$$

On the other hand, it is easy to obtain that

$$
I\left(u_{n}\right)-\frac{l_{1}}{2 l_{2}} I^{\prime}\left(u_{n}\right) u_{n} \leq C+C\left\|u_{n}\right\| .
$$

Then, from above, we can have

$$
\|u\|^{2} \leq C+C\left\|u_{n}\right\|,
$$

which contradicts $\left\|u_{n}\right\| \rightarrow+\infty$. Therefore $\left\{u_{n}\right\}$ is bounded in $\mathbf{H}$.

Case II. if $\int_{\Omega}\left|\Delta u_{n}\right|^{2} d x \rightarrow+\infty$. By (H1), let $l_{2}=\max \left\{1, m^{\prime}\right\}$, we also can obtain that $\left\{u_{n}\right\}$ is bounded in $\mathbf{H}$.

This lemma is completely proved.

Lemma 2.3. Suppose that (H1)-(H5) hold, then we have

(1) there exist constants $\rho>0, \alpha>0$ such that $\left.I\right|_{\partial B_{\rho}} \geq \alpha$ with $B_{p}=\{u \in \mathbf{H}\|u\| \leq \rho\}$;

(2) $I\left(t \phi_{1}\right) \rightarrow-\infty$ as $t \rightarrow+\infty$.

Proof. By (H1)-(H4), we see that for any $\varepsilon>0$, there exist constants $C_{1}>0, C_{2}$ such that for all $(x, s) \in \Omega \times R$, one have

$$
F(x, s) \leq \frac{1}{2} \varepsilon s^{2}+C_{1} s^{p+1}
$$

Choosing $\varepsilon>0$ small enough, we have

$$
\begin{aligned}
I(u) & =\frac{1}{2} \int_{\Omega}|\Delta u|^{2} d x+\frac{1}{2} \widehat{M}\left(\int_{\Omega}|\nabla u|^{2} d x\right)-\int_{\Omega} F(x, u) d x \\
& \geq \frac{1}{2} \int_{\Omega}|\Delta u|^{2} d x+\frac{1}{2} m_{0} \int_{\Omega}|\nabla u|^{2} d x-\int_{\Omega} F(x, u) d x \\
& \geq \frac{1}{2} l_{1}\|u\|^{2}-\frac{\varepsilon}{2}\|u\|_{L^{2}}^{2}-C_{1}\|u\|_{L^{p+1}}^{p+1} \\
& \geq \frac{1}{2}\left(l_{1}-\varepsilon\right)\|u\|^{2}-C_{3}\|u\|^{p+1} .
\end{aligned}
$$


by (3), (5), (7) and the Sobolev inequality. So, part 1 is proved if we choose $\|u\| \rho>0$ small enough.

On the other hand, we have

$$
\begin{aligned}
I(u) & =\frac{1}{2} \int_{\Omega}|\Delta u|^{2} d x+\frac{1}{2} \widehat{M}\left(\int_{\Omega}|\nabla u|^{2} d x\right)-\int_{\Omega} F(x, u) d x \\
& \leq \frac{1}{2} \int_{\Omega}|\Delta u|^{2} d x+\frac{1}{2} m_{1} \int_{\Omega}|\nabla u|^{2} d x-\int_{\Omega} F(x, u) d x \\
& \geq \frac{1}{2} l_{2}\|u\|^{2}-\|u\|_{\Theta}^{\Theta}+C_{4} .
\end{aligned}
$$

using (4) and (H5). Hence,

$$
I\left(t \varphi_{1}\right) \leq \frac{1}{2} l_{2} t^{2}\left\|\varphi_{1}\right\|^{2}-t^{\Theta}\left\|\varphi_{1}\right\|_{\Theta}^{\Theta}+C_{4} \rightarrow-\infty
$$

as $t \rightarrow+\infty$ and part 2 is proved.

Proof of Theorem 2.1. From Lemmas 2.2 and 2.3, it is clear to see that $I(u)$ satisfies the hypotheses of Lemma 1.1. Therefore $I(u)$ has a critical point.

\section{Existence result II}

Theorem 3.1. Assume that (H1) holds. In addition, assume the following conditions are hold:

(H6) $f(x, t) t \geq 0$ for $x \in \Omega, t \in R$;

(H7) $\lim _{t \rightarrow 0} \frac{f(x, t)}{t}=\alpha, \lim _{|t| \rightarrow+\infty} \frac{f(x, t)}{t}=\beta$, uniformly in a.e $x \in \Omega$, where $\frac{\alpha}{\min \left\{1, m_{0}\right\}}<\lambda_{1}\left(\lambda_{1}+m^{\prime}\right)<\beta<+\infty$.

Then Equation (1) has at least two nontrivial solutions, one of which is positive and the other is negative.

Let $u^{+}=\max \{u, 0\}, u^{-}=\min \{u, 0\}$. Consider the following problem

$$
\begin{cases}\Delta^{2} u-M\left(\int_{\Omega}|\nabla u|^{2} d x\right) \Delta u=f^{+}(x, u), & \text { in } \Omega, \\ u=\Delta u=0, & \text { on } \partial \Omega,\end{cases}
$$

where

$$
f^{+}(x, t)= \begin{cases}f(x, t) & \text { if } t \geq 0 \\ 0, & \text { if } t<0\end{cases}
$$

Define the corresponding functional $I^{+}: \mathbf{H} \rightarrow R$ as follows:

$$
I^{+}(u)=\frac{1}{2} \int_{\Omega}|\Delta u|^{2} d x+\frac{1}{2} \widehat{M}\left(\int_{\Omega}|\nabla u|^{2} d x\right)-\int_{\Omega} F^{+}(x, u) d x, \quad \forall u \in \mathbf{H},
$$

where $F^{+}(x, u)=\int_{0}^{u} f^{+}(x, t) d t$. Obviously, $I^{+} \in C^{\mathrm{l}}(\mathbf{H}, R)$. Let $u$ be a critical point of $I^{+}$ which implies that $u$ is the weak solution of Equation (8). Futhermore, by the weak maximum principle it follows that $u \geq 0$ in $\Omega$. Thus $u$ is also a solution of Equation (1). 
Similarly, we also can define

$$
f^{-}(x, t)= \begin{cases}f(x, t) & \text { if } t \leq 0 \\ 0, & \text { if } t<0\end{cases}
$$

and

$$
I^{-}(u)=\frac{1}{2} \int_{\Omega}|\Delta u|^{2} d x+\frac{1}{2} \widehat{M}\left(\int_{\Omega}|\nabla u|^{2} d x\right)-\int_{\Omega} F^{-}(x, u) d x, \quad \forall u \in \mathbf{H},
$$

where $F^{-}(x, u)=\int_{0}^{u} f^{-}(x, t) d t$. Obviously, $I^{-} \in C^{1}(\mathbf{H}, R)$. Let $u$ be a critical point of $I^{-}$ which implies that $u$ is the weak solution of Equation (1) with $I^{-}(u)=I(u)$.

Lemma 3.2. Assume that (H1), (H6), and (H7) hold, then $I^{ \pm}$satisfies the (PS) condition.

Proof. We just prove the case of $I^{+}$. The arguments for the case of $I^{-}$are similar. Since $\Omega$ is bounded and (H7) holds, then if $\left\{u_{n}\right\}$ is bounded in $\mathbf{H}$, by using the Sobolve embedding and the standard procedures, we can get a convergent subsequence. So we need only to show that $\left\{u_{n}\right\}$ is bounded in $\mathbf{H}$.

Let $\left\{u_{n}\right\} \subset \mathbf{H}$ be a sequence such that

$$
I^{+}\left(u_{n}\right) \rightarrow c, \quad \nabla I^{+}\left(u_{n}\right) \rightarrow 0 .
$$

By $(\mathrm{H} 7)$, it is easy to see that

$$
\left|f^{+}(x, s) s\right| \leq C\left(1+|s|^{2}\right) .
$$

Now, (9) implies that, for all $\varphi \in \mathbf{H}$, we have

$$
\int_{\Omega} \Delta u_{n} \Delta \phi d x+M\left(\int_{\Omega}\left|\nabla u_{n}\right|^{2} d x\right) \int_{\Omega} \nabla u_{n} \nabla \phi d x=\int_{\Omega} f^{+}\left(x, u_{n}\right) \phi d x \rightarrow 0 .
$$

Set $\varphi=u_{n}$, we have

$$
\begin{aligned}
\min \left\{1, m_{0}\right\}\left\|u_{n}\right\|^{2} & \leq \int_{\Omega}\left|\Delta u_{n}\right|^{2} d x+M\left(\int_{\Omega}\left|\nabla u_{n}\right|^{2} d x\right) \int_{\Omega}\left|\nabla u_{n}\right|^{2} d x \\
& =\int_{\Omega} f^{+}\left(x, u_{n}\right) u_{n} d x+\left\langle\nabla I^{+}\left(u_{n}\right), u_{n}\right\rangle \\
& \leq \int_{\Omega} f^{+}\left(x, u_{n}\right) u_{n} d x+o(1)\left\|u_{n}\right\| \\
& \leq C+C|| u_{n}\left\|_{L^{2}}^{2}+o(1)\right\| u_{n} \| .
\end{aligned}
$$

Next, we will show that $\left\|u_{n}\right\|_{L^{2}}^{2}$ is bounded. If not, we may assume that $\left\|u_{n}\right\|_{L} 2 \rightarrow$ $+\infty$ as $n \rightarrow+\infty$. Let $\omega_{n}=\frac{u_{n}}{\left\|u_{n}\right\|_{L^{2}}}$, then $\left\|\omega_{n}\right\|_{L^{2}}=1$. From (11), we have

$$
\left\|\omega_{n}\right\|^{2} \leq o(1)+C+\frac{o(1)}{\left\|u_{n}\right\|_{L^{2}}} \frac{\left\|u_{n}\right\|}{\left\|u_{n}\right\|_{L^{2}}}=o(1)+C+o(1)\left\|\omega_{n}\right\|,
$$


thus $\left\{\omega_{n}\right\}$ is bounded in $\mathbf{H}$. Passing to a subsequence, we may assume that there exists $\omega \in \mathbf{H}$ with $\|\omega\|_{L^{2}}=1$ such that

$$
\begin{gathered}
\omega_{n} \rightarrow \omega, \quad \text { weakly in } \mathbf{H}, \quad n \rightarrow+\infty, \\
\omega_{n} \rightarrow \omega, \quad \text { strongly in } L^{2}(\Omega), \quad n \rightarrow+\infty .
\end{gathered}
$$

On the other hand, $\left\|u_{n}\right\|_{L^{2}} \rightarrow+\infty$ as $n \rightarrow+\infty$, by Poincare inequality, it is easy to know that $\int_{\Omega}\left|\Delta u_{n}\right|^{2} d x \rightarrow+\infty$ as $n \rightarrow+\infty$. Thus by (H1), the function $M\left(\int_{\Omega}\left|\nabla u_{n}\right|^{2} d x\right)=m^{\prime}$. So as $n \rightarrow+\infty$, by (10), we have

$$
\int_{\Omega} \Delta \omega \Delta \phi d x+m^{\prime} \int_{\Omega} \nabla \omega \nabla \phi d x \int_{\Omega} \beta \omega^{+} \phi d x=0, \quad \forall \phi \in \mathbf{H} .
$$

Then $\omega \in \mathbf{H}$ is a weak solution of the equation

$$
\Delta^{2} \omega-m^{\prime} \Delta \omega=\beta \omega^{+} .
$$

The weak maximum principle implies that $\omega=\omega^{+} \geq 0$. Choosing $\varphi(x)=\phi_{1}(x)>0$, which is the corresponding eigenfunctions of $\lambda_{1}$. From (10), we get

$$
\int_{\Omega} \Delta \omega \Delta \varphi_{1} d x+m^{\prime} \int_{\Omega} \nabla \omega \nabla \varphi_{1} d x=\beta \int_{\Omega} \omega^{+} \varphi_{1} d x .
$$

On the other hand, we can easily see that $\Lambda=\lambda_{1}\left(\lambda_{1}+m^{\prime}\right)$ is the eigenvalue of the problem

$$
\begin{cases}\Delta^{2} u+m^{\prime} \Delta u=\Lambda u, & \text { in } \Omega, \\ \Delta u=u=0, & \text { on } \partial \Omega\end{cases}
$$

and the corresponding eigenfunction is still $\phi_{1}(x)$. If $\omega(x)>0$, we also have

$$
\int_{\Omega} \Delta \omega \Delta \varphi_{1} d x+m^{\prime} \int_{\Omega} \nabla \omega \nabla \varphi_{1} d x=\Lambda \int_{\Omega} \omega^{+} \varphi_{1} d x,
$$

which follows that $\omega \equiv 0$ by $\Lambda<\beta$ But this conclusion contradicts $\|\omega\|_{L^{2}}=1$.

Hence $\left\{u_{n}\right\}$ is bounded in $\mathbf{H}$.

Now we prove that the functionals $I^{ \pm}$has a mountain pass geometry.

Lemma 3.3. Assume that (H1), (H7) hold, then we have

(1) there exists $\rho, R>0$ such that $I^{ \pm}(u)>R$, if $\|u\|=\rho$;

(2) $I^{ \pm}(u)$ are unbounded from below.

Proof. By (H7), for any $\varepsilon>0$, there exists $C_{1}>0, C_{2}>0$ such that $\forall(x, s) \in \Omega \times R$, we have

$$
F(x, s) \leq \frac{1}{2}(\alpha+\varepsilon) s^{2}+C_{1} s^{p+1}
$$

and

$$
F(x, s) \geq \frac{1}{2}(\beta+\varepsilon) s^{2}+C_{2}
$$

where $2<p<2 *=\left\{\begin{array}{l}\frac{2 N}{N-2} N>2, \\ +\infty \quad N \leq 2 .\end{array}\right.$ 
We just prove the case of $I^{+}$. The arguments for the case of $I^{-}$are similar. Let $\varphi=$ $t \phi_{1}$. When $t$ is sufficiently large, by (16) and (H1), it is easy to see that

$$
\begin{aligned}
I^{+}\left(t \varphi_{1}\right) & =\frac{1}{2} \int_{\Omega}\left|\Delta\left(t \varphi_{1}\right)\right|^{2} d x+\frac{1}{2} \widehat{M}\left(\int_{\Omega}\left|\nabla\left(t \varphi_{1}\right)\right|^{2} d x\right)-\int_{\Omega} F^{+}\left(x, t \varphi_{1}\right) d x \\
& \leq \frac{1}{2} \int_{\Omega}\left|\Delta\left(t \varphi_{1}\right)\right|^{2} d x+\frac{1}{2} m^{\prime} \int_{\Omega}\left|\nabla\left(t \varphi_{1}\right)\right|^{2} d x-\int_{\Omega} \frac{1}{2}(\beta-\varepsilon)\left(t \varphi_{1}\right)^{2}-C_{2} d x \\
& =\frac{t^{2}}{2}\left[\int_{\Omega}\left|\Delta \varphi_{1}\right|^{2} d x+m^{\prime} \int_{\Omega}\left|\Delta \varphi_{1}\right|^{2} d x-(\beta-\varepsilon) \int_{\Omega} \varphi_{1}^{2} d x\right]+C_{2}|\Omega| \\
& =\frac{t^{2}}{2}[\Lambda-(\beta-\varepsilon)]|| \varphi_{1} \|_{L^{2}}+C_{2}|\Omega| \\
& \rightarrow-\infty, \text { as } t \rightarrow+\infty .
\end{aligned}
$$

On the other hand, by (17), (H1), the Poincare inequality and the Sobolve embedding, we have

$$
\begin{aligned}
I^{+}(u) & =\frac{1}{2} \int_{\Omega}|\Delta u|^{2} d x+\frac{1}{2} \widehat{M}\left(\int_{\Omega}|\nabla u|^{2} d x\right)-\int_{\Omega} F^{+}(x, u) d x \\
& \geq \frac{1}{2} \min \left\{1, m_{0}\right\}|| u \|-\frac{\alpha+\varepsilon}{2} \int_{\Omega}|u|^{2} d x-C_{1} \int_{\Omega}|u|^{p+1} d x \\
& \geq\left(\frac{1}{2} \min \left\{1, m_{0}\right\}-\frac{\alpha+\varepsilon}{2 \Lambda}\right)\|u\|-C_{4}\|u\|^{p+1}
\end{aligned}
$$

where $C_{4}$ is a constant. Choosing $\|u\|=\rho$ small enough, we can obtain $I^{+}(u) \geq R>0$ if $\|u\|=\rho$.

Proof of Theorem 3.1. From Lemma 3.3, it is easy to see that there exists $e \in \mathbf{H}$ with $\|e\|>\rho$ such that $I^{ \pm}(e)<0$.

Define

$$
P=\{\gamma:[0,1] \rightarrow H: \gamma \text { is continuous and } \gamma(0)=0, \gamma(1)=e\},
$$

and

$$
c^{ \pm}=\inf _{\gamma \in P} \max _{t \in[0,1]} I^{ \pm}(\gamma(t)) .
$$

From Lemma 3.3, we have

$$
I^{ \pm}(0)=0, \quad I^{ \pm}(e)<0,\left.\quad I^{ \pm}(u)\right|_{\partial B_{\rho}} \geq R>0 .
$$

Moreover, by Lemma 3.2, the functions $I^{ \pm}$satisfies the (PS)-condition. By Lemma 1.1, we know that $c^{+}$is a critical value of $I^{+}$and there is at least one nontrivial critical point in $\mathbf{H}$ corresponding to this value. This critical in nonnegative, then the strong maximum principle implies that is a positive solution of Equation (1). By an analogous way we know there exists at least one negative solution, which is a nontrivial critical point of $I^{-}$Hence, Equation (1) admits at least a positive solution and a negative solution. 


\section{Author details}

${ }^{1}$ College of Science, Hohai University, Nanjing, 210098, P. R. China ${ }^{2}$ Department of Mathematics, Nanjing University of Aeronautics and Astronautics, Nanjing 210016, P. R. China

\section{Authors' contributions}

In this manuscript the authors studied the existence and multiplicity of solutions for an interesting fourth-order elliptic equation by using the famous mountain pass lemma. Moreover, in this work, the authors' supplements done in [123]. All authors typed, read and approved the final manuscript.

\section{Competing interests}

The authors declare that they have no competing interests.

Received: 26 August 2011 Accepted: 17 January 2012 Published: 17 January 2012

\section{References}

1. Ma, TF: Existence results for a model of nonlinear beam on elastic bearings. Appl. Math Lett. 13, 11-15 (2000). doi:10.1016/50893-9659(00)00026-4

2. Ma, TF: Existence results and numerical solutions for a beam equation with nonlinear boundary conditions. Appl Numer Math. 47, 189-196 (2003). doi:10.1016/S0168-9274(03)00065-5

3. Ma, TF: Positive solutions for a nonlocal fourth-order equations of Kirchhoff type. Discrete Contin Dyn Syst (suppl). 694-703 (2007)

4. An, Y, Liu, R: Existence of nontrivial solutions of an asymptotically linear fourth-order elliptic equations. Nonlinear Anal. 68, 3325-3331 (2008). doi:10.1016/.jna.2007.03.028

5. Bernis, F, Azorero, JG, Peral, I: Existence and multiplicity of nontrivial solutions in semilinear critical problems of fourthorder. Adv Diff Equ. 1, 219-240 (1996)

6. Chen, Y, McKenna, PJ: Traveling waves in a nonlinear suspension beam: the-oretical results and numerical observations. J Diff Equ. 135, 325-355 (1997)

7. Liu, X, Huang, Y: On sign-changing solution for a fourth-order asymptotically linear elliptic problem. Nonlinear Anal. 72 , 2271-2276 (2010). doi:10.1016/j.na.2009.11.001

8. Lazer, AC, McKenna, PJ: Large-amplitude periodic oscillations in suspension bridge: some new connections with nonlinear analysis. SIAM Rev. 32, 537-578 (1990). doi:10.1137/1032120

9. McKenna, PJ, Walter, W: Traveling waves in a suspension bridge. SIAM J Appl Math. 50, 703-715 (1990). doi:10.1137/ 0150041

10. Pei, R: Multiple solutions for biharmonic equations with asymptotically linear nonlinearities. Bound Value Probl 2010, Article ID 241518. 11 (2010)

doi:10.1186/1687-2770-2012-6

Cite this article as: Wang and An: Existence and multiplicity of solutions for a fourth-order elliptic equation. Boundary Value Problems 2012 2012:6.

\section{Submit your manuscript to a SpringerOpen ${ }^{\circ}$ journal and benefit from:}

- Convenient online submission

- Rigorous peer review

- Immediate publication on acceptance

- Open access: articles freely available online

- High visibility within the field

- Retaining the copyright to your article

Submit your next manuscript at $\boldsymbol{\wedge}$ springeropen.com 